\title{
Substrate-Assisted Cysteine Deprotonation in the Mechanism of Dimethylargininase (DDAH) from Pseudomonas aeruginosa ${ }^{\dagger}$
}

\author{
Everett M. Stone, Alison L. Costello, David L. Tierney, and Walter Fast
}

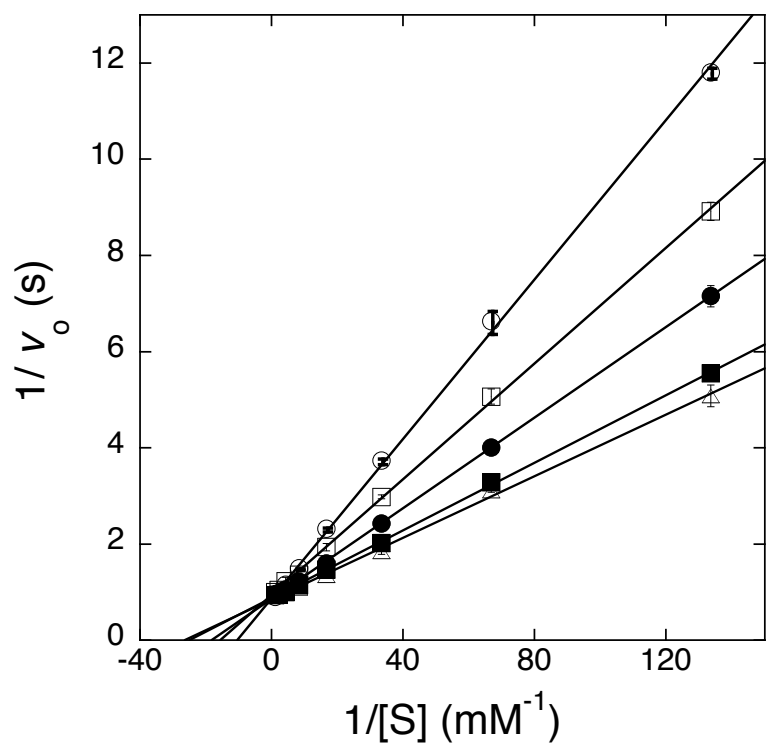

Figure S1. Competitive inhibition of wild-type Pa DDAH by L-lysine. For display purposes, a double-reciprocal plot is shown for inhibition of $S$-methyl-L-thiocitrulline hydrolysis by $1.25(\Delta), 2.5$ $(\square), 5(\bullet), 10(\square)$, and $15(\bigcirc) \mathrm{mM}$ L-lysine. Non-linear fits for competitive inhibition indicate a $K_{\mathrm{i}}$ value of $4.0 \pm 0.3 \mathrm{mM}$ at $\mathrm{pH}$ 7.3. See materials and methods for experimental details.

Substrate protects against inactivation by iodoacetamide.. In order to determine whether inactivation of $P a$ DDAH by iodoacetmide occurs by modification at the active-site, a substrateprotection experiment was carried out. Solutions of $P a$ DDAH $(14 \mu \mathrm{M})$ in Tris buffer $(250 \mathrm{mM})$ and $\mathrm{KCl}(250 \mathrm{mM})$ at $\mathrm{pH} 8.5$, were co-incubated with either iodoacetamide $(1 \mathrm{mM})$ alone, or with iodoacetamide $(1 \mathrm{mM})$ and $N^{\omega}$-methyl-L-arginine $(2.5 \mathrm{mM})$. As a control, $\mathrm{Pa}$ DDAH was incubated in buffer alone. At time points between 0 and $6 \mathrm{~min}$, aliquots of the incubation mixtures were 
diluted 26-fold into a buffer containing potassium phosphate (100 mM), EDTA (1 mM), 5, 5'dithiobis-(2-nitrobenzoic acid) $(180 \mu \mathrm{M})$, and $S$-methyl-L-thiocitrulline $(1 \mathrm{mM})$ and assayed as described previously $(S 1)$. Observed rates were plotted as a percent of the original activity against time and fit to a single exponential equation (Figure S2). The observed rate of $\mathrm{Pa}$ DDAH inactivation by iodoacetamide was significantly slowed by co-incubation with the substrate, suggesting that inactivation occurs through modification at the active-site.

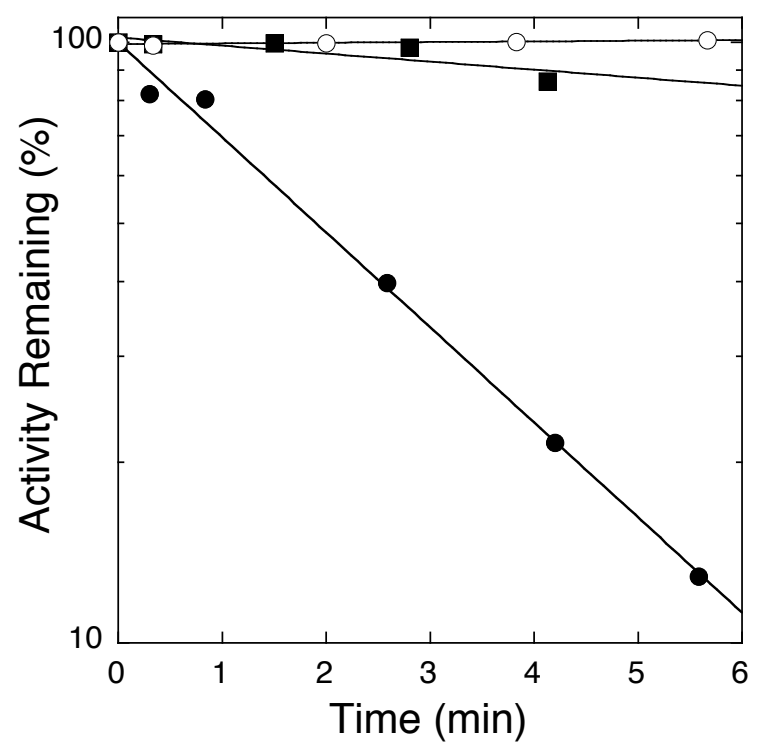

Figure S2. Substrate protects against $\mathrm{Pa}$ DDAH inactivation by iodoacetamide. Control incubations of Pa DDAH (open circles) show no loss of activity. Incubations with iodoacetamide (closed circles) show time-dependent inactivation. Incubations with iodoacetamide and $N^{\omega}$-methylL-arginine (closed squares) show that substrate protects against inactivation.

(S1) Stone, E. M., and Fast, W. (2005) A continuous spectrophotometric assay for dimethylarginine dimethylaminohydrolase. Anal Biochem 343, 335-7. 